\title{
Tissue morphogenesis mediated by the Arabidopsis receptor kinase STRUBBELIG involves a clathrin-dependent process
}

Jin $\mathrm{Gao}^{1}$, Ajeet Chaudhary ${ }^{1}$, Prasad Vaddepalli ${ }^{1,2}$, Marie-Kristin Nagel $^{3}$, Erika Isono ${ }^{3}$, and Kay

Schneitz ${ }^{*}$

${ }^{1}$ Entwicklungsbiologie der Pflanzen, Wissenschaftszentrum Weihenstephan, Technische Universität München, Freising, Germany

${ }^{2}$ Present address: Laboratory of Biochemistry, Wageningen University, Wageningen, the Netherlands

${ }^{3}$ Department of Biology, Chair of Plant Physiology and Biochemistry, University of Konstanz,

Konstanz, Germany

${ }^{*}$ Corresponding author

Entwicklungsbiologie der Pflanzen

Wissenschaftszentrum Weihenstephan

Technische Universität München

Emil-Ramann-Str. 4

D-85354 Freising

Email:kay.schneitz@tum.de

Tel: +49 8161715438

Email addresses authors:

Jin Gao: gao@wzw.tum.de;

Ajeet Chaudhary: ajeet.chaudhary@tum.de

Prasad Vaddepalli: prasad.vaddepalli@wur.nl

Marie-Kristin Nagel: marie-kristin.nagel@uni-konstanz.de

Erika Isono: erika.isono@uni-konstanz.de

Date of submission: 19.10.2018

Number of tables and figures: 2 tables, 7 figures.

Word count: 5380

Supplementary data: 2 figures, 1 table

Running title: clathrin and SUB signaling

Key words: clathrin, endocytosis, endomembrane, plants, receptor kinase, STRUBBELIG, tissue morphogenesis, vesicular trafficking 


\title{
Highlight
}

The Arabidopsis receptor kinase STRUBBELIG is internalized by clathrin-mediated endocytosis and affects clathrin-dependent processes in a tissue-dependent manner.

\begin{abstract}
Signaling mediated by cell surface receptor kinases is central to the coordination of growth patterns during organogenesis. Receptor kinase signaling is in part controlled through endocytosis and subcellular distribution of the respective receptor kinase. For the majority of plant cell surface receptors the underlying trafficking mechanisms are not characterized. In Arabidopsis, tissue morphogenesis relies on the atypical receptor kinase STRUBBELIG (SUB). Here, we approach the endocytic mechanism of SUB. Our data reveal that a functional SUB:EGFP fusion is ubiquitinated in vivo. We further show that plasma membrane-bound SUB:EGFP becomes internalized in a clathrin-dependent fashion. We also find that SUB:EGFP associates with the transGolgi network and accumulates in multivesicular bodies and the vacuole. Coimmunoprecipitation experiments reveal that SUB:EGFP and clathrin are present within the same protein complex. Our genetic analysis shows that $S U B$ and CLATHRIN HEAVY CHAIN 2 promote root hair patterning. By contrast, SUB behaves as a negative regulator of a clathrin-dependent process during floral development. Taken together, the data indicate that SUB undergoes clathrin-mediated endocytosis, that this process does not dependent on stimulation of SUB signaling by an exogenous agent, and that $S U B$ genetically interacts with clathrin-dependent pathways in a tissuespecific manner.
\end{abstract}

\section{Introduction}

Intercellular communication is a central requirement for tissue morphogenesis as cells have to orchestrate their relative behavior to allow proper organ development. Cell surface receptor kinases play crucial roles in this process. Control of tissue morphogenesis in Arabidopsis thaliana involves the leucine-rich repeat (LRR) receptor kinase (RK) STRUBBELIG (SUB). SUB, also known as SCRAMBLED (SCM), controls several developmental processes, including floral morphogenesis, integument outgrowth, leaf development and root hair patterning (Chevalier et al., 2005; Kwak et al., 2005; Lin et al., 2012). SUB represents an atypical receptor kinase as enzymatic activity of its kinase domain is not required for its function in vivo 
(Chevalier et al., 2005; Vaddepalli et al., 2011). SUB is glycosylated in the endoplasmic reticulum (ER) (Hüttner et al., 2014), subject to ER-associated protein degradation (Vaddepalli et al., 2011; Hüttner et al., 2014), and found at the plasma membrane (PM) (Yadav et al., 2008; Vaddepalli et al., 2014). SUB not only localizes to the PM but is also present at plasmodesmata (PD), channels interconnecting most plant cells (Otero et al., 2016; Sager and Lee, 2018), where it physically interacts with the PD-specific protein QUIRKY (QKY) (Vaddepalli et al., 2014). In line with a function in RK-mediated control of PD-based intercellular communication SUB and QKY function in a non-cell-autonomous manner (Yadav et al., 2008; Vaddepalli et al., 2014) indicating that SUB signaling involves a yet unknown factor that moves between cells. More recently, a genetic link of SUB signaling to cell wall biology has also been put forward as the cell wall-localized $\beta-1,3$ glucanase ZERZAUST (ZET) participates in SUB signal transduction and sub, qky and zet mutants share overlapping defects in cell wall biochemistry (Fulton et al., 2009; Vaddepalli et al., 2017).

The maintenance of the PM composition is in part achieved through exocytosis/secretion and endocytosis (Paez Valencia et al., 2016; Reynolds et al., 2018). In general plant cells internalize PM-bound material or cargo via membrane transport into the trans-Golgi network (TGN), an organelle that also functions as an early endosome (EE) and that serves as a sorting hub for subsequent trafficking pathways. The cargo may get recycled back to the PM via secretory vesicles. Cargo may also be destined to degradation via endosomal transport to multivesicular bodies (MVBs), also known as late endosomes (LEs), containing intra-luminal vesicles (ILVs). MVBs eventually fuse with the tonoplast discharging their content into the vacuolar lumen where it becomes degraded.

Internalization of PM proteins is mediated by clathrin-dependent and clathrinindependent endocytosis (Geldner and Robatzek, 2008; Robinson et al., 2008; Irani and Russinova, 2009; Paez Valencia et al., 2016; Reynolds et al., 2018). Clathrinmediated endocytosis (CME) is a central mechanism for the internalization of PMlocalized material or cargo (Dhonukshe et al., 2007; Paez Valencia et al., 2016; Reynolds et al., 2018). CME involves the budding of cargo-containing clathrin-coated vesicles (CCVs) from the PM. CCVs consist of vesicles surrounded by a polyhedral 
lattice of clathrin triskelia being made of three clathrin heavy chains (CHCs), each bound by a clathrin light chain (CLC) (Fotin et al., 2004). In Arabidopsis, three genes encode CLC chains while the likely redundant acting $\mathrm{CHC1}$ and $\mathrm{CHC} 2$ encode clathrin heavy chains (Scheele and Holstein, 2002). Clathrin is also present at the TGN/EE, at a subpopulation of MVB/LEs, and at the cell plate indicating that it functions in multiple vesicular trafficking steps, and cytokinesis in the plant cell (Samuels et al., 1995; Staehelin and Moore, 1995; Konopka et al., 2008; Fujimoto et al., 2010; Stierhof and El Kasmi, 2010; Kang et al., 2011; Van Damme et al., 2011; Ito et al., 2012).

Fine-tuning the spatio-temporal dynamics of receptor-mediated endocytosis and endosomal trafficking is a central element in the regulation of RK-dependent signal transduction. Such a mechanism can for example maintain the steady-state level of RKs at the PM through recycling internalized RKs back to the PM, promote signaling by activated RK complexes localized on endosomes, or attenuate RK signaling by controlled removal of activated receptors from the PM followed by sorting into MVBs and finally degradation in the vacuole (Geldner and Robatzek, 2008; Irani and Russinova, 2009; Di Rubbo and Russinova, 2012; Bakker et al., 2017; Critchley et al., 2018).

Following their internalization and subsequent trafficking upon RK stimulation by exogenous application of ligand has been instrumental in analyzing the endocytic pathways of several plant RKs, including BRASSINOSTEROID INSENSITIVE 1 (BRI1) (Russinova et al., 2004; Geldner et al., 2007; Irani et al., 2012; Di Rubbo et al., 2013), FLAGELLIN SENSING 2 (FLS2) (Robatzek et al., 2006; Beck et al., 2012; Du et al., 2013; Mbengue et al., 2016), or PEP1 RECEPTOR 1 (PEPR1) (OrtizMorea et al., 2016). No ligand for SUB has been described to date rendering such an experimental strategy currently impossible. However, some RKs undergo endocytosis independently of exogenous application of ligand, including BRI1 (Russinova et al., 2004; Geldner et al., 2007; Jaillais et al., 2008), SOMATIC EMBRYOGENESIS RECEPTOR-LIKE KINASE 1 (SERK1) (Shah et al., 2001; Shah et al., 2002), BRI1ASSOCIATED RECEPTOR KINASE 1 (BAK1)/SERK3 (Russinova et al., 2004), and ARABIDOPSIS CRINKLY4 (ACR4) (Gifford et al., 2005). 
SUB can be found in internal compartments as well (Kwak and Schiefelbein, 2008; Yadav et al., 2008; Vaddepalli et al., 2011; Wang et al., 2016b) and it was recently shown that ovules of plants homozygous for a hypomorphic allele of HAPLESS13 (HAP13) preferentially accumulate signal from a functional SUB:EGFP reporter in the cytoplasm, rather than the PM (Wang et al., 2016b). HAP13/AP1M2 encodes the $\mu 1$ subunit of the adaptor protein (AP) complex AP1 that is present at the TGN/EE network and is involved in post-Golgi vesicular trafficking to the PM, vacuole and cell-division plane (Park et al., 2013; Teh et al., 2013; Wang et al., 2013). Interestingly, ovules of plants with reduced HAP13/AP1M2 activity show sub-like integuments (Wang et al., 2016b). These results indicate that the AP1 complex is involved in subcellular distribution of SUB in a functionally relevant manner.

Here, we have further assessed the internalization and subsequent endocytic trafficking behavior of SUB. We show that the intracellular domain of a functional SUB:EGFP fusion protein becomes ubiquitinated. Upon endocytosis SUB:EGFP is sorted to MVBs and the vacuole. Our data further indicate that CME contributes to internalization of SUB:EGFP. Finally, we provide genetic data suggesting that $C H C$ is part of the $S U B$-dependent signaling mechanism that mediates root hair patterning while $S U B$ is a negative regulator of a $C H C$-dependent pathway in floral tissue.

\section{Materials and methods}

Plant work, plant genetics and plant transformation

Arabidopsis thaliana (L.) Heynh. var. Columbia (Col-0) and var. Landsberg (erecta mutant) (Ler) were used as wild-type strains. Plants were grown as described earlier (Fulton et al., 2009). The sub-1 (Ler) was described previously (Chevalier et al., 2005). The sub-9 mutant (Col), carrying a T-DNA insertion (SAIL_1158_D09), was described in (Vaddepalli et al., 2011). The chcl-1 (SALK_112213), chcl-2 (SALK_103252), chc2-1 (SALK_028826) and chc2-2 (SALK_042321) alleles (all Col) (Alonso et al., 2003) were described in (Kitakura et al., 2011). Wild-type and mutant plants were transformed with different constructs using Agrobacterium strain GV3101/pMP90 (Koncz and Schell, 1986) and the floral dip method (Clough and Bent, 1998). Transgenic T1 plants were selected on Kanamycin $(50 \mu \mathrm{g} / \mathrm{ml})$, Hygromycin $(20 \mu \mathrm{g} / \mathrm{ml})$ or Glufosinate (Basta) $(10 \mu \mathrm{g} / \mathrm{ml})$ plates and transferred to soil for further inspection. The hydroxytamoxifen-inducible line INTAM $>>$ RFP- 
HUB/Col line (HUB) was described previously (Robert et al., 2010; Kitakura et al., 2011). Seedlings were grown on half-strength Murashige and Skook (1/2 MS) agar plates (Murashige and Skoog, 1962).

\section{Recombinant DNA work}

For DNA and RNA work standard molecular biology techniques were used. PCRfragments used for cloning were obtained using Q5 high-fidelity DNA polymerase (New England Biolabs, Frankfurt, Germany). All PCR-based constructs were sequenced. Primer sequences used in this work are listed in supplementary material Table SX.

\section{Reporter constructs}

The pCAMBIA2300-based pSUB::SUB:EGFP construct was described previously (Vaddepalli et al., 2011). To obtain pUBQ10::SUB:EGFP, a $2 \mathrm{~kb}$ promoter fragment of UBQ10 (At4g05320) was amplified from Ler genomic DNA using primers pUBQ(KpnI)_F and pUBQ(AscI)_R. The resulting PCR product was digested using $\mathrm{KpnI} / \mathrm{AscI}$ and used to replace the pSUB fragment in pSUB::SUB:EGFP. The pGL2::GUS:EGFP construct was assembled using the GreenGate system (Lampropoulos et al., 2013). The promoter region of GL2 (AT1G79840) was amplified with primer pGL2_F1 and pGL2_R1 from genomic Col-0 DNA. The internal BsaI site was removed during the procedure as described in (Lampropoulos et $a l .$, 2013). The GUS coding sequence was amplified from plasmid pBI121 (Jefferson et al., 1987) with primer GUS_F and GUS_R, digested with BsaI and used for further cloning.

\section{Genotyping PCR}

PCR-based genotyping was performed with the following primer combinations: $s u b$ -

9, SUB_LP158, SUB_RP158, and SAIL_LB2; chc2 salk-042321, CHC2-LP321, CHC2-RP321, and SALK_LBb1.3; chc2 salk-028826, CHC2_LP826, CHC2_RP826, and SALK_LBb1.3; chc1 salk-112213,CHC1_LP213,CHC1_RP213, and SALK_LBb1.3; chc1 salk-103252, CHC1_LP252,CHC1_RP252, and SALK_LBb1.3.

\section{Chemical treatments}


Brefeldin A (BFA), cycloheximide, tyrphostin A23 (TyrA23), Wortmannin, Concanamycin A (ConcA) were obtained from Sigma-Aldrich and used from stock solutions in DMSO (50 mM BFA, cycloheximide, TyrA23; 30 mM Wortmannin, 2 $\mathrm{mM}$ ConcA). FM4-64 was purchased from Molecular Probes (2 $\mathrm{mM}$ stock solution in water). Five day-old seedlings were incubated for the indicated times in liquid 1/2 MS medium containing $50 \mu \mathrm{M}$ BFA, $50 \mu \mathrm{M}$ cycloheximide, $75 \mu \mathrm{M}$ TyrA23, $33 \mu \mathrm{M}$ Wortmannin, and $2 \mu \mathrm{M}$ ConcA. For FM4-64 staining seedlings were incubated in 4 $\mu \mathrm{M}$ FM4-64 in liquid 1/2 MS medium for 5 minutes prior to imaging. 4hydroxytamoxifen was obtained from Sigma-Aldrich (10 mM stock solution in ethanol). Seedlings were grown for 3 days on $1 / 2 \mathrm{MS}$ plates, transferred onto $1 / 2 \mathrm{MS}$ plates containing $2 \mu \mathrm{M}$ 4-hydroxytamoxifen (or ethanol as mock treatment) for four days and then imaged using confocal microscopy.

\section{Immunoprecipitation and western blot analysis}

$500 \mathrm{mg}$ of 7-day wild-type or transgenic seedlings were lysed using a TissueLyser II (Qiagen) and homogenized in $1 \mathrm{ml}$ lysis buffer A (50mM Tris- $\mathrm{HCl} \mathrm{pH} 7.5,100 \mathrm{mM}$ $\mathrm{NaCl}, 0.1 \mathrm{mM}$ PMSF, $0.5 \%$ Triton X-100, protease inhibitor mixture (Roche)). Cell lysate was mildly agitated for 15 min on ice and centrifuged for 15 minutes at 13000 g. For lines carrying GFP-tagged proteins supernatant was incubated with GFPTRAP_MA magnetic agarose beads (ChromoTek) for 2 hours at $4^{\circ} \mathrm{C}$. Beads were concentrated using a magnetic separation rack. Samples were washed four times in buffer B (50mM Tris-HCl pH7.5, $100 \mathrm{mM} \mathrm{NaCl,} 0.1 \mathrm{mM}$ PMSF, 0.2\% Triton X-100, protease inhibitor mixture (Roche)). Bound proteins were eluted from beads by heating the samples in $30 \mu 12 x$ Laemmli buffer for 5 minutes. Samples were separated by SDS-PAGE and analyzed by immunoblotting according to standard protocols. Primary antibodies included mouse monoclonal anti-GFP antibody 3E6 (Invitrogen/Thermo Fisher Scientific), mouse monoclonal anti-ubiquitin antibody P4D1 (Santa Cruz Biotechnology), and polyclonal anti-CHC antibody AS10 690-ALP (Agrisera). Secondary antibodies were obtained from Pierce/ThermoFisher Scientific: goat anti-rabbit IgG antibody (1858415) and goat anti-mouse IgG antibody (1858413).

\section{Microscopy and art work}


To assess the cellular structure of floral meristems samples were stained with mPS-PI (Truernit et al., 2008). Confocal laser scanning microscopy was performed with an Olympus FV1000 setup using an inverted IX81 stand and FluoView software (FV10ASW version 01.04.00.09) (Olympus Europa GmbH, Hamburg, Germany) equipped with a water-corrected 40x objective (NA 0.9) at 3x digital zoom. For SUB:EGFP subcellular localization upon drug treatments or colocalization with endosomal markers confocal laser scanning microscopy was performed on epidermal cells of root meristems located about eight to 12 cells above the quiescent center using a Leica TCS SP8 X microscope equipped with GaAsP (HyD) detectors. The following objectives were used: a water-corrected $63 \mathrm{x}$ objective (NA 1.2), a 40x objective (NA 1.1), and a 20x immersion objective (NA 0.75). Scan speed was set at $400 \mathrm{~Hz}$, line average at between 2 and 4, and the digital zoom at 4.5 (colocalization with FM4-64), 3 (drug treatments) or 1 (root hair patterning). EGFP fluorescence excitation was done at $488 \mathrm{~nm}$ using a multi-line argon laser ( 3 percent intensity) and detected at 502 to $536 \mathrm{~nm}$. FM4-64 fluorescence was excited using a $561 \mathrm{~nm}$ laser (1 percent intensity) and detected at 610 to $672 \mathrm{~nm}$. For the direct comparisons of fluorescence intensities, laser, pinhole, and gain settings of the confocal microscope were kept identical when capturing the images from the seedlings of different treatments. The intensities of fluorescence signals at the PM were quantified using Leica LAS X software (version 3.3.0.16799). For the measurement of the fluorescence levels at the PM optimal optical sections of root cells were used for measurements. On the captured images the fluorescent circumference of an individual cell (ROI, region of interest) was selected with the polygon tool. The mean pixel intensity readings for the selected ROIs were recorded and the average values were calculated. For determination of colocalization, the distance from the center of each EGFP spot to the center of the nearest FM4-64, $\mathrm{mKO}$ or mRFP signal was measured by hand on single optical sections using ImageJ/Fiji software (Schindelin et al., 2012). If the distance between two puncta was below the resolution limit of the objectives lens $(0.24 \mu \mathrm{m})$ the signals were considered to colocalize (Ito et al., 2012). Arabidopsis seedlings were covered with a $22 \times 22 \mathrm{~mm}$ glass cover slip of 0.17 mm thickness (No. 1.5H, Paul Marienfeld GmbH \& Co. KG, Lauda-Königshofen, Germany). Scanning electron microscopy was performed essentially as reported previously (Schneitz et al., 1997; Sieburth and Meyerowitz, 1997). Images were adjusted for color and contrast using ImageJ/Fiji software. 


\section{Results}

The endocytic route of SUB:EGFP

To investigate the endocytic pathway followed by SUB we made use of a previously well-characterized line carrying the $s u b-1$ null allele and a transgene encoding a SUB:EGFP translational fusion driven by its endogenous promoter (pSUB::SUB:EGFP). The line exhibits a wild-type phenotype demonstrating the presence of a functional reporter (Vaddepalli et al., 2011; Vaddepalli et al., 2014). We studied the subcellular distribution of the pSUB::SUB:EGFP reporter signal in epidermal cells of the root meristem using confocal laser scanning microscopy. These cells serve as an ideal model as $S U B$ promotes the early patterning of root hairs, cells that are generated by the epidermis (Dolan et al., 1993). In the absence of any obvious exogeneous stimulation of SUB signaling we observed SUB:EGFP signal at the PM and in cytoplasmic foci (Fig. 1A). Moreover, we noticed that the SUB:EGFP signal labelled structures resembling vesicles as well as the vacuole. These observations raise the possibility that SUB:EGFP undergoes internalization from the PM and is shuttled to the vacuole for degradation.

To assess the early process of SUB:EGFP endocytosis we imaged cells upon a 5minutes treatment with the endocytic tracer dye FM4-64 (Fig. 1A,D). Using a previously described criterion for colocalization (Ito et al., 2012) the internal SUB:EGFP and FM4-64 signals were considered colocalized when the distance between the centers of the two types of signals was below the limit of resolution of the objective, in our case $0.24 \mu \mathrm{m}$. We observed that 70 percent $(\mathrm{n}=344)$ of all cytoplasmic SUB:EGFP foci were also marked by FM4-64 supporting endocytosis of SUB:EGFP. To further investigate internalization of SUB:EGFP we treated five daysold seedlings with Wortmannin. Wortmannin is a phosphatidylphosphate-3-kinase inhibitor that among others interferes with vesicle formation from the PM (Tse et al., 2004; Wang et al., 2009; Ito et al., 2012; Cui et al., 2016). We analyzed the number of internal SUB:EGFP-labelled puncta in cells upon treatment with $33 \mu \mathrm{M}$ Wortmannin for 60 minutes. We found a substantial reduction in the number of such puncta in drug-treated cells when compared to mock-treated cells (Fig. 2A). Moreover, we noted a significant increase in SUB:EGFP signal intensity at the PM in Wortmannin-treated cells. 
To explore if endosomal trafficking of SUB:EGFP involves the TGN/EE we investigated colocalization of SUB:EGFP with the TGN marker mRFP:SYP43 (Ebine et al., 2011; Ito et al., 2012; Uemura et al., 2012) (Fig. 1B,D). We observed a frequency of 44 percent colocalization $(n=278)$ between internal SUB:EGFP and RFP:SYP43 puncta. To further assess colocalization of SUB:EGFP with the TGN we made use of a previously characterized translational fusion between CLC2 and monomeric Kushiba Orange under the control of the cauliflower mosaic virus 35S promoter (mKO:CLC) (Fujimoto et al., 2010). CLC2 fused to fluorescent tags also localizes to the TGN in live cell imaging experiments (Ito et al., 2012). We observed a frequency of 33 percent colocalization $(n=365)$ between internal SUB:EGFP and mKO:CLC puncta (Fig. 1C,D). To corroborate the presence of SUB:EGFP at the TGN/EE we exposed $s u b-1$ pSUB::SUB:EGFP seedlings to the fungal toxin Brefeldin A (BFA). Treatment with BFA results in the formation of so-called BFA compartments or bodies that contain secretory and endocytic vesicles (Robinson et al., 2008; Paez Valencia et al., 2016). We observed prominent SUB:EGFP signal in BFA compartments in root epidermal cells of seedlings treated with DMSO for 30 minutes followed by a DMSO/BFA $(50 \mu \mathrm{M})$ treatment for 60 minutes, confirming previous data (Fig. 2B) (Kwak and Schiefelbein, 2008; Yadav et al., 2008; Vaddepalli et al., 2011; Wang et al., 2016b).

To explore the relative contribution of signal at the TGN/EE originating from the secretion of newly translated SUB:EGFP versus endocytic SUB:EGFP-derived signal we first treated seedlings with the protein synthesis inhibitor cycloheximide $(50 \mu \mathrm{M})$ for 60 minutes followed by co-incubation with $50 \mu \mathrm{M}$ BFA for 30 minutes. In those seedlings SUB:EGFP still prominently localized to BFA bodies (Fig. 2C) as noted earlier (Wang et al., 2016a). Taken together, the results indicate that a large fraction of SUB:EGFP in BFA bodies originated from the PM.

We next investigated if internalized SUB:EGFP is sorted into MVBs. Apart from affecting vesicle formation at the PM Wortmannin also interferes with the maturation of LEs and causes formation of enlarged MVB/LEs (Tse et al., 2004; Wang et al., 2009; Cui et al., 2016). Treating seedlings for 60 minutes with $33 \mu \mathrm{M}$ wortmannin results in the formation of large globular structures labelled by SUB:EGFP signal (Fig. 2A). Such structures are typical for enlarged MVBs (Jia et al., 2013). In 
accordance with these results SUB:EGFP was detected at MVBs in immunogold electron microscopy experiments (Vaddepalli et al., 2014).

Concanamycin A (ConcA) inhibits vacuolar ATPase activity at the TGN/EE and in the tonoplast thereby interfering with the trafficking of newly synthesized materials to the PM, the transport of cargo from the TGN/EE to the vacuole, and the vacuolar degradation of cargo (Dettmer et al., 2006; Robinson et al., 2008; Viotti et al., 2010; Scheuring et al., 2011). Upon treatment with $2 \mu \mathrm{M}$ ConcA for 1 hour seedlings showed large roundish structures labelled by a diffuse SUB:EGFP signal (Fig. 2D) indicating that SUB:EGFP was not degraded efficiently and thus accumulated in the vacuole.

Taken together the results are consistent with the notion that the endocytic route of SUB:EGFP involves the TGN/EE, the MVB/LEs, and the vacuole where it becomes degraded. A noticeable portion of SUB:EGFP puncta colocalizes with the TGN/EE, supporting passage of SUB:EGFP through the TGN/EE. However, we cannot exclude that a fraction of SUB:EGFP also traffics via an TGN/EE-independent route, as does for example the AtPep1-PEPR1 signaling complex (Ortiz-Morea et al., 2016).

\section{SUB:EGFP is ubiquitinated in vivo}

Ubiquitination plays an important role in endocytosis and endosomal sorting of PM proteins (MacGurn et al., 2012; Paez Valencia et al., 2016; Isono and Kalinowska, 2017), such as the brassinosteroid receptor BRI1 (Martins et al., 2015) or the auxin efflux facilitator PINFORMED 2 (PIN2) (Leitner et al., 2012). To test if SUB:EGFP is ubiquitinated in vivo we made use of our $s u b-1$ pSUB::SUB:EGFP reporter line as well as a previously described line carrying the SUB:EGFP translation fusion driven by the UBIQUITIN10 (UBQ) promoter (pUBQ::SUB:EGFP) (Vaddepalli et al., 2017). We immunoprecipitated SUB:EGFP from seven days-old, plate-grown seedlings using an anti-GFP antibody. Immunoprecipitates were subsequently probed with the commonly used P4D1 anti-ubiquitin antibody recognizing mono- and polyubiquitinated proteins. P4D1-dependent signal could not be reproducibly detected when testing immunoprecipitates from lines expressing the pSUB::SUB:EGFP reporter due to low abundance of SUB:EGFP in the immunoprecipitate. By contrast, we clearly observed a high-molecular weight smear in immunoprecipitates obtained 
from pUBQ::SUB:EGFP lines (Fig. 3). This smear is typical for ubiquitinated proteins. We did not detect signals in immunoprecipitates obtained from wild-type seedlings. The results indicate that a fraction of SUB proteins becomes ubiquitinated.

\section{SUB:EGFP internalization involves clathrin-mediated endocytosis}

So far, the obtained results indicate that SUB:EGFP is continuously internalized and eventually targeted to the vacuole for degradation. Next we wanted to assess if SUB:EGFP relates to a clathrin-dependent process. We first tested if SUB:EGFP and endogenous $\mathrm{CHC}$ occur in the same complex in vivo. To this end we immunoprecipitated SUB:EGFP from seven days-old, plate-grown pUBQ::SUB:EGFP sub-1 seedlings using an anti-GFP antibody. Immunoprecipitates were subsequently probed using an anti-CHC antibody. We could detect a CHCsignal in immunoprecipitates derived from SUB:EGFP plants but not from wild-type (Fig. 4) indicating that SUB:EGFP and CHC are present in the same protein complex in vivo.

Next we assessed the contribution of clathrin to the internalization and subcellular distribution of SUB:EGFP. To this end, we investigated the effects of a transient but robust impairment of clathrin activity on the internalization and subcellular distribution of SUB:EGFP. Ectopic expression of the C-terminal part of $\mathrm{CHC} 1$ (HUB1) results in a dominant-negative effect due to the HUB1 fragment binding to and out-titrating clathrin light chains (Liu et al., 1995). To assess the effect of the presence of the HUB fragment on the subcellular distribution of SUB:EGFP we crossed a previously characterized 4-hydroxytamoxifen-inducible INTAM $>>$ RFPCHC1 (HUB) line (Robert et al., 2010; Kitakura et al., 2011) into a Col-0 wild-type line carrying the pUBQ::SUB:EGFP reporter. We then analyzed epidermal cells of the root meristem of HUB/pUBQ::SUB:EGFP plants, hemizygous for each transgene, upon induction.

We first determined the length of induction period that enabled us to detect by confocal microscopy a defect in endocytosis, as indicated by a reduction of internal FM4-64 foci following a 5 to 10 minutes exposure to the stain. Under our growth conditions a significant reduction of internal FM4-64 puncta was observed after three days of continuous growth on induction medium while near complete absence of 
internal FM4-64 foci was detected after four days (Fig. 5B,C). If SUB:EGFP participates in CME a block in HUB-sensitive endocytosis should result in fewer internal SUB:EGFP-labelled foci and higher SUB:EGFP signal at the PM when compared to the SUB:EGFP-derived signal of a control line. We found a significant reduction in cytoplasmic SUB:EGFP puncta in the HUB/pUBQ::SUB:EGFP line after three days of growth on induction medium in comparison to the control (Fig. 5B). Upon four days of induction we detected an increase in SUB:EGFP signal at the PM (Fig. 5C). Taken together, our results suggest that CME contributes to the internalization of SUB:EGFP.

\section{SUB genetically interacts with CLATHRIN HEAVY CHAIN}

To further assess the role of clathrin in the SUB signaling mechanism we tested a possible genetic interaction between $S U B$ and $C H C$. To this end we made use of several previously characterized T-DNA insertion lines carrying knock-out alleles of CHC1 and CHC2 (Kitakura et al., 2011). Plants lacking CHC1 as well as CHC2 function appear to be lethal (Kitakura et al., 2011). However, mutations in individual CHC genes result in endocytosis defects and affect for example polar distribution of PIN proteins, internalization of ATRBOHD, stomatal movement, and resistance to powdery mildew (Kitakura et al., 2011; Hao et al., 2014; Wu et al., 2015; Larson et $a l ., 2017)$.

To test if clathrin is involved in $S U B$-controlled processes we first investigated if $c h c 1$ and $c h c 2$ mutants show a defect in root hair patterning. To this end we generated homozygous $c h c$ mutants carrying a translational fusion of bacterial $ß$-glucuronidase (GUS) to EGFP (GUS:EGFP) under the control of the Arabidopsis GLABRA2 (GL2) promoter (pGL2::GUS:EGFP). The GL2 promoter drives expression specifically in non-root hair cells and is commonly used to monitor root hair patterning (Masucci et al., 1996; Kwak et al., 2005). Interestingly, we found that all chc alleles tested showed root hair patterning defects similar to $s u b-9$ with $c h c 2$ alleles causing more prominent aberrations compared to $c h c 1$ mutations (Fig. 6, Supplementary Fig. S1). In addition, $c h c 1$ sub-9 or chc 2 sub- 9 double mutants did not show an obviously exacerbated phenotype indicating that $\mathrm{CHC1}, \mathrm{CHC} 2$ and $S U B$ do not act in an additive fashion. Thus, the results indicate that $\mathrm{CHCl}$ and $\mathrm{CHC} 2$ promote root hair pattern formation and that they function in the same genetic pathway than $S U B$. 
Next, we assessed if $C H C 1$ and $C H C 2$ participate in $S U B$-dependent floral development. In the Col-0 background null alleles of SUB cause a weaker floral phenotype when compared to similar alleles in the Ler background (Vaddepalli et al., 2011). Still, the $s u b-9$ allele causes mild silique twisting, mis-oriented cell division plants in the L2 layer of floral meristems, and ovule defects (Fig. 7) (Tables 1 and 2) (Vaddepalli et al., 2011). By contrast, we did not detect any obvious defects in floral meristems, flowers and ovules of plants homozygous for the tested chc1 or chc2 alleles (Fig. 7, Supplementary Fig. S2) (Tables 1 and 2). We then investigated the phenotype of $c h c 1$ sub- 9 and $c h c 2$ sub-9 double mutants. Interestingly, the cell division defects in the L2 layer of the FM were reduced in $c h c 1$ sub-9 and chc2 sub-9 double mutants in comparison to sub-9 single mutants (Fig. 7, Supplementary Fig. S2) (Table 1). Suppression of the sub-9 phenotype in $c h c 1 s u b-9$ or chc2-sub-9 double mutants was also observed for silique twisting and ovule development (Fig. 7, Supplementary Fig. S2) (Table 2). The results suggest that $S U B$ is a negative genetic regulator of $\mathrm{CHCl}$ and $\mathrm{CHC} 2$ function in floral meristem, ovule and silique development.

\section{Discussion}

An impressive body of published work has elucidated many of the intricacies of receptor-mediated endocytosis of plant RKs. Much is known about the internalization and endocytic trafficking of plant RKs with functional kinase domains. The atypical RK SUB carries an inconspicuous kinase domain, however, enzymatic kinase activity could not be demonstrated in in vitro biochemical experiments and is not required for its function in vivo (Chevalier et al., 2005; Vaddepalli et al., 2011; Kwak et al., 2014). Using SUB as a model we have explored the endocytic route of an atypical RK. We investigated this process by examining the subcellular distribution of a functional SUB:EGFP reporter in epidermal cells of the root meristem. Our data are compatible with the notion that PM-localized SUB becomes internalized and traffics from the TGN/EE to MVB/LEs and eventually the vacuole where it is destined for degradation. SUB:EGFP was observed to enter this endocytic route in the apparent absence of activation of SUB signaling by artificial stimulation or application of exogenous ligand. A similar observation was for example made for ACR4 (Gifford et $a l ., 2005)$. One interpretation of this finding could be that endogenous SUB ligand is 
always present in sufficient levels to promote SUB endocytosis. In another possible scenario, the rate of SUB internalization may be independent from ligand availability as was shown for BRI1 (Russinova et al., 2004; Geldner et al., 2007). In any case, our data indicate that the endocytic route of the atypical RK SUB for the most part seems to adhere to the established pattern of plant receptor-mediated endocytosis.

Apart from being a central signal for proteasome-mediated degradation ubiquitination is a major endocytosis determinant of PM proteins (Haglund and Dikic, 2012; Isono and Kalinowska, 2017). Several plant RKs are known to be ubiquitinated, including FLS2 (Lu et al., 2011), BRI1 (Martins et al., 2015; Zhou et al., 2018), and LYK5 (Liao et al., 2017). The observed in vivo ubiquitination of SUB:EGFP is compatible with the notion of SUB being internalized and transported to the vacuole for degradation. However, it remains to be determined which E3 ubiquitin ligase promotes ubiquitination of SUB and how SUB endocytosis relates to the control of its signaling. Internalization can be linked with downstream responses, as was demonstrated for FLS2 or the AtPep1-PEPR complex (Mbengue et al., 2016; OrtizMorea et al., 2016), or contribute to signal downregulation, as it is the case for BRI1 (Irani et al., 2012; Zhou et al., 2018) or LYK5 (Liao et al., 2017).

Several lines of evidence support the notion of SUB:EGFP undergoing CME. First, CHC in vivo co-immunoprecipitated with SUB:EGFP. Second, we observed a reduction in intra-cellular SUB:EGFP puncta accompanied with a stronger SUB:EGFP signal at the PM in the HUB-line upon induction. Third, our genetic analysis revealed a connection of SUB with a clathrin-dependent process. Plants with a defect in $\mathrm{CHC} 2$ show significantly reduced endocytosis rate of FM4-64 and aberrant polar localization of the polar auxin transporter PINFORMED 1 (PIN1) (Kitakura et al., 2011) as well as reduced internalization of, for example, PEP1 \{Ortiz-Morea et al., 2016, \#6940\}, FLS2 (Mbengue et al., 2016), and BRI1 (Wang et al., 2015). Accordingly, chc2 mutants show multiple defects, including patterning defects in the embryo (Kitakura et al., 2011), impaired mitogen-activated protein kinase (MAPK) activation (Ortiz-Morea et al., 2016), and defective stomatal closure and callose deposition upon bacterial infection (Mbengue et al., 2016). Our genetic analysis revealed that $\mathrm{CHC2}$, and to a lesser effect $\mathrm{CHC1}$, also affects root hair patterning. 
Importantly, it provides evidence for a biologically relevant interaction between $S U B$ and a $\mathrm{CHC}$-dependent process.

Interestingly, the data suggest that the type of genetic interaction between $S U B$ and $C H C$ depends on the tissue context. In the root, $S U B$ and $C H C$ promote root hair patterning. Several hypotheses are conceivable that could explain the result. As our data support the notion of SUB:EGFP undergoing CME, one model states that CME of SUB is required for root hair patterning. Therefore, SUB internalization in single chc mutants would be reduced resulting in a hyperaccumulation of SUB at the PM. Two alternative further scenarios are compatible with this notion. In the first scenario hyperaccumulation of SUB at the PM interferes with root hair patterning. This view is supported by the observation that not just a reduction of $S U B$ activity but also ectopic expression of $S U B$ in $p 35 S:: S U B$ plants results in a weak defect in root hair patterning (Kwak and Schiefelbein, 2007), similar to what we observed for chc2 mutants. In the second scenario, a reduction of SUB internalization leads to fewer SUB-labelled endosomes, which in turn impairs root hair patterning. This scenario implies that SUB signals while being present on endosomes. In another model, a reduction of $\mathrm{CHC}$ activity could influence clathrin-dependent secretion of newly translated and/or recycled SUB to the PM thereby reducing the level of active SUB at the PM below a certain threshold. Finally, given the pleiotropic phenotype of $c h c$ mutants the genetic data do not rule out a more indirect interaction between $S U B$ and $C H C$. Further work remains to be done to discriminate between the different possibilities. However, we currently favor the notion that CME of SUB is critical for root hair patterning as a block of CME of SUB:EGFP in the HUB line results in a reduction of internalized SUB:EGFP vesicles and elevated levels of SUB:EGFP at the PM.

In contrast to the positive genetic role of $S U B$ and $C H C$ in root hair patterning the apparent wild-type appearance of floral organs of sub chc double mutants indicates that $S U B$ is a negative regulator of a $C H C$-dependent process during floral development. The molecular mechanism remains to be investigated. CME could for instance promote the internalization of a PM-resident signaling molecule, thereby attenuating its activity. This endocytic process could be counteracted upon by SUB. For example, in a sub mutant the activity of the hypothetical signaling factor at the PM is reduced through increased endocytosis. In a sub chc double mutant the 
principally higher level of internalization caused by the lack of SUB activity is offset by reduced $\mathrm{CME}$ due to impaired $\mathrm{CHC}$ function. Thus, the individual $s u b$ and $c h c$ effects cancel each other out in sub chc double mutants and the respective plants show flowers with apparent wild-type morphology. It will be an exciting challenge to unravel the molecular details of how SUB and clathrin interact to allow tissue morphogenesis in future studies.

\section{Supplementary data}

Supplementary Figure S1: Comparison of the root hair patterning phenotype of wild type, sub-9, chc1-1 and chc2-1 mutants.

Supplementary Figure S2: Comparison of the floral phenotype of wild type, sub-9, chc1-1 and chc2-1 mutants.

Supplementary Table S1: List of all primers used in this study.

\section{Acknowledgements}

We thank Jiří Friml for the INTAM $>>$ RFP-CHC1 (HUB) line, Masaru Fujimoto for the mKO:CLC2 reporter and Tomohiro Uemura for the mRFP:SYP43 line. We also thank Silke Robatzek for critical reading of the manuscript. This work was funded by the German Research Council (DFG) through grant SFB924 (TP A06) to EI and SFB924 (TP A02) to KS.

\section{References}

Alonso JM, Stepanova AN, Leisse TJ, Kim CJ, Chen H, Shinn P, Stevenson DK, Zimmerman J, Barajas P, Cheuk R et al. 2003. Genome-wide insertional mutagenesis of Arabidopsis thaliana. Science 301, 653-657.

Bakker J, Spits M, Neefjes J, Berlin I. 2017. The EGFR odyssey - from activation to destruction in space and time. J Cell Sci 130, 4087-4096.

Beck M, Zhou J, Faulkner C, MacLean D, Robatzek S. 2012. Spatio-temporal cellular dynamics of the Arabidopsis flagellin receptor reveal activation statusdependent endosomal sorting. Plant Cell 24, 4205-4219. 


\section{Chevalier D, Batoux M, Fulton L, Pfister K, Yadav RK, Schellenberg M,}

Schneitz K. 2005. STRUBBELIG defines a receptor kinase-mediated signaling pathway regulating organ development in Arabidopsis. Proc. Natl. Acad. Sci. U S A 102, 9074-9079.

Clough SJ, Bent AF. 1998. Floral dip: a simplified method for Agrobacteriummediated transformation of Arabidopsis thaliana. Plant J 16, 735-743.

Critchley WR, Pellet-Many C, Ringham-Terry B, Harrison MA, Zachary IC, Ponnambalam S. 2018. Receptor tyrosine kinase ubiquitination and de-ubiquitination in signal transduction and receptor trafficking. Cells 7 ,

Cui Y, Shen J, Gao C, Zhuang X, Wang J, Jiang L. 2016. Biogenesis of plant prevacuolar multivesicular bodies. Mol Plant 9, 774-786.

Dettmer J, Hong-Hermesdorf A, Stierhof YD, Schumacher K. 2006. Vacuolar $\mathrm{H}+$-ATPase activity is required for endocytic and secretory trafficking in Arabidopsis. Plant Cell 18, 715-730.

Dhonukshe P, Aniento F, Hwang I, Robinson DG, Mravec J, Stierhof YD, Friml J. 2007. Clathrin-mediated constitutive endocytosis of PIN auxin efflux carriers in Arabidopsis. Curr Biol 17, 520-527.

Di Rubbo S, Irani NG, Kim SY, Xu ZY, Gadeyne A, Dejonghe W, Vanhoutte I, Persiau G, Eeckhout D, Simon S et al. 2013. The clathrin adaptor complex AP-2 mediates endocytosis of BRASSINOSTEROID INSENSITIVE1 in Arabidopsis. Plant Cell 25, 2986-2997.

Di Rubbo S, Russinova E. 2012. Receptor-mediated endocytosis in plants. In: Šamaj J, ed. Plant Endocytosis. Heidelberg:Springer, 151-164.

Dolan L, Janmaat K, Willemsen V, Linstead P, Poethig S, Roberts K, Scheres B. 1993. Cellular organisation of the Arabidopsis thaliana root. Development 119, 71-84. Du Y, Tejos R, Beck M, Himschoot E, Li H, Robatzek S, Vanneste S, Friml J. 2013. Salicylic acid interferes with clathrin-mediated endocytic protein trafficking. Proc Natl Acad Sci U S A 110, 7946-7951.

Ebine K, Fujimoto M, Okatani Y, Nishiyama T, Goh T, Ito E, Dainobu T, Nishitani A, Uemura T, Sato MH et al. 2011. A membrane trafficking pathway regulated by the plant-specific RAB GTPase ARA6. Nat Cell Biol 13, 853-859. Fotin A, Cheng Y, Sliz P, Grigorieff N, Harrison SC, Kirchhausen T, Walz T. 2004. Molecular model for a complete clathrin lattice from electron cryomicroscopy. Nature 432, 573-579. 
Fujimoto M, Arimura S, Ueda T, Takanashi H, Hayashi Y, Nakano A, Tsutsumi N. 2010. Arabidopsis dynamin-related proteins DRP2B and DRP1A participate together in clathrin-coated vesicle formation during endocytosis. Proc Natl Acad Sci U S A 107, 6094-6099.

Fulton L, Batoux M, Vaddepalli P, Yadav RK, Busch W, Andersen SU, Jeong S, Lohmann JU, Schneitz K. 2009. DETORQUEO, QUIRKY, and ZERZAUST represent novel components involved in organ development mediated by the receptorlike kinase STRUBBELIG in Arabidopsis thaliana. PLoS Genet 5, e1000355.

Geldner N, Hyman DL, Wang X, Schumacher K, Chory J. 2007. Endosomal signaling of plant steroid receptor kinase BRI1. Genes Dev 21, 1598-1602.

Geldner N, Robatzek S. 2008. Plant receptors go endosomal: a moving view on signal transduction. Plant Physiol 147, 1565-1574.

Gifford ML, Robertson FC, Soares DC, Ingram GC. 2005. ARABIDOPSIS CRINKLY4 function, internalization, and turnover are dependent on the extracellular crinkly repeat domain. Plant Cell 17, 1154-1166.

Haglund K, Dikic I. 2012. The role of ubiquitylation in receptor endocytosis and endosomal sorting. J Cell Sci 125, 265-275.

Hao H, Fan L, Chen T, Li R, Li X, He Q, Botella MA, Lin J. 2014. Clathrin and membrane microdomains cooperatively regulate $\mathrm{RbohD}$ dynamics and activity in Arabidopsis. Plant Cell 26, 1729-1745.

\section{Hüttner S, Veit C, Vavra U, Schoberer J, Dicker M, Maresch D, Altmann F,} Strasser R. 2014. A context-independent N-glycan signal targets the misfolded extracellular domain of Arabidopsis STRUBBELIG to endoplasmic-reticulumassociated degradation. Biochem J 464, 401-411.

Irani NG, Russinova E. 2009. Receptor endocytosis and signaling in plants. Current Opinion in Plant Biology 12, 653-659.

Irani NG, Di Rubbo S, Mylle E, Van den Begin J, Schneider-Pizoń J, Hniliková J, Šíša M, Buyst D, Vilarrasa-Blasi J, Szatmári AM et al. 2012. Fluorescent castasterone reveals BRI1 signaling from the plasma membrane. Nat Chem Biol 8 , 583-589.

Isono E, Kalinowska K. 2017. ESCRT-dependent degradation of ubiquitylated plasma membrane proteins in plants. Curr Opin Plant Biol 40, 49-55. 
Ito E, Fujimoto M, Ebine K, Uemura T, Ueda T, Nakano A. 2012. Dynamic behavior of clathrin in Arabidopsis thaliana unveiled by live imaging. Plant J 69, 204216.

Jaillais Y, Fobis-Loisy I, Miège C, Gaude T. 2008. Evidence for a sorting endosome in Arabidopsis root cells. Plant J 53, 237-247.

Jefferson RA, Kavanagh TA, Bevan MW. 1987. GUS fusions: ß-glucuronidase as a sensitive and veratile gene fusion marker in higher plants. EMBO Journal 6, 39013908.

Jia T, Gao C, Cui Y, Wang J, Ding Y, Cai Y, Ueda T, Nakano A, Jiang L. 2013. ARA7(Q69L) expression in transgenic Arabidopsis cells induces the formation of enlarged multivesicular bodies. J Exp Bot 64, 2817-2829.

Kang BH, Nielsen E, Preuss ML, Mastronarde D, Staehelin LA. 2011. Electron tomography of RabA4b- and PI-4K $\beta 1$-labeled trans Golgi network compartments in Arabidopsis. Traffic 12, 313-329.

Kitakura S, Vanneste S, Robert S, Lofke C, Teichmann T, Tanaka H, Friml J. 2011. Clathrin mediates endocytosis and polar distribution of PIN auxin transporters in Arabidopsis. Plant Cell 23, 1920-1931.

Koncz C, Schell J. 1986. The promoter of TL-DNA gene 5 controls the tissuespecific expression of chimaeric genes carried by a novel Agrobacterium binary vector. Mol. Gen. Genet. 204, 383-396.

Konopka CA, Backues SK, Bednarek SY. 2008. Dynamics of Arabidopsis dynamin-related protein $1 \mathrm{C}$ and a clathrin light chain at the plasma membrane. Plant Cell 20, 1363-1380.

Kwak SH, Schiefelbein J. 2007. The role of the SCRAMBLED receptor-like kinase in patterning the Arabidopsis root epidermis. Dev Biol 302, 118-131.

Kwak SH, Schiefelbein J. 2008. A feedback mechanism controlling SCRAMBLED receptor accumulation and cell-type pattern in Arabidopsis. Curr Biol 18, 1949-1954. Kwak SH, Shen R, Schiefelbein J. 2005. Positional signaling mediated by a receptor-like kinase in Arabidopsis. Science 307, 1111-1113.

Kwak SH, Woo S, Lee MM, Schiefelbein J. 2014. Distinct signaling mechanisms in multiple developmental pathways by the SCRAMBLED receptor of Arabidopsis. Plant Physiol 166, 976-987. 


\section{Lampropoulos A, Sutikovic Z, Wenzl C, Maegele I, Lohmann JU, Forner J.}

2013. GreenGate - A novel, versatile, and efficient cloning system for plant transgenesis. PLoS One 8, e83043.

Larson ER, Van Zelm E, Roux C, Marion-Poll A, Blatt MR. 2017. Clathrin heavy chain subunits coordinate endo- and exocytic traffic and affect stomatal movement. Plant Physiol 175, 708-720.

Leitner J, Petrášek J, Tomanov K, Retzer K, Pařezová M, Korbei B, Bachmair A, Zažímalová E, Luschnig C. 2012. Lysine63-linked ubiquitylation of PIN2 auxin carrier protein governs hormonally controlled adaptation of Arabidopsis root growth. Proc Natl Acad Sci U S A 109, 8322-8327.

Liao D, Cao Y, Sun X, Espinoza C, Nguyen CT, Liang Y, Stacey G. 2017. Arabidopsis E3 ubiquitin ligase PLANT U-BOX13 (PUB13) regulates chitin receptor LYSIN MOTIF RECEPTOR KINASE5 (LYK5) protein abundance. New Phytol 214, 1646-1656.

Lin L, Zhong SH, Cui XF, Li J, He ZH. 2012. Characterization of temperaturesensitive mutants reveals a role for receptor-like kinase SCRAMBLED/STRUBBELIG in coordinating cell proliferation and differentiation during Arabidopsis leaf development. Plant J 72, 707-720.

Liu SH, Wong ML, Craik CS, Brodsky FM. 1995. Regulation of clathrin assembly and trimerization defined using recombinant triskelion hubs. Cell 83, 257-267.

Lu D, Lin W, Gao X, Wu S, Cheng C, Avila J, Heese A, Devarenne TP, He P, Shan L. 2011. Direct ubiquitination of pattern recognition receptor FLS2 attenuates plant innate immunity. Science 332, 1439-1442.

MacGurn JA, Hsu PC, Emr SD. 2012. Ubiquitin and membrane protein turnover: from cradle to grave. Annu Rev Biochem 81, 231-259.

Martins S, Dohmann EM, Cayrel A, Johnson A, Fischer W, Pojer F, SatiatJeunemaître B, Jaillais Y, Chory J, Geldner N et al. 2015. Internalization and vacuolar targeting of the brassinosteroid hormone receptor BRI1 are regulated by ubiquitination. Nat Commun 6, 6151.

Masucci JD, Rerie WG, Foreman DR, Zhang M, Galway ME, Marks MD, Schiefelbein JW. 1996. The homeobox gene GLABRA2 is required for positiondependent cell differentiation in the root epidermis of Arabidopsis thaliana.

Development 122, 1253-1260. 
Mbengue M, Bourdais G, Gervasi F, Beck M, Zhou J, Spallek T, Bartels S, Boller T, Ueda T, Kuhn H et al. 2016. Clathrin-dependent endocytosis is required for immunity mediated by pattern recognition receptor kinases. Proc Natl Acad Sci U S A 113, 11034-11039.

Murashige T, Skoog F. 1962. A revised medium for a rapid growth and bioassays with tobacco tissue cultures. Physiologia Plantarum 15, 473-497.

Ortiz-Morea FA, Savatin DV, Dejonghe W, Kumar R, Luo Y, Adamowski M, Van den Begin J, Dressano K, Pereira de Oliveira G, Zhao X et al. 2016. Dangerassociated peptide signaling in Arabidopsis requires clathrin. Proc Natl Acad Sci U S A 113, 11028-11033.

Otero S, Helariutta Y, Benitez-Alfonso Y. 2016. Symplastic communication in organ formation and tissue patterning. Curr Opin Plant Biol 29, 21-28.

Paez Valencia J, Goodman K, Otegui MS. 2016. Endocytosis and endosomal trafficking in plants. Annu Rev Plant Biol 67, 309-335.

Park M, Song K, Reichardt I, Kim H, Mayer U, Stierhof YD, Hwang I, Jurgens

G. 2013. Arabidopsis mu-adaptin subunit AP1M of adaptor protein complex 1 mediates late secretory and vacuolar traffic and is required for growth. Proc Natl Acad Sci U S A 110, 10318-10323.

Reynolds GD, Wang C, Pan J, Bednarek SY. 2018. Inroads into internalization: five years of endocytic exploration. Plant Physiol 176, 208-218.

Robatzek S, Chinchilla D, Boller T. 2006. Ligand-induced endocytosis of the pattern recognition receptor FLS2 in Arabidopsis. Genes Dev 20, 537-542.

Robert S, Kleine-Vehn J, Barbez E, Sauer M, Paciorek T, Baster P, Vanneste S, Zhang J, Simon S, Covanova M et al. 2010. ABP1 mediates auxin inhibition of clathrin-dependent endocytosis in Arabidopsis. Cell 143, 111-121.

Robinson DG, Jiang L, Schumacher K. 2008. The endosomal system of plants: charting new and familiar territories. Plant Physiol 147, 1482-1492.

Russinova E, Borst JW, Kwaaitaal M, Caño-Delgado A, Yin Y, Chory J, de Vries SC. 2004. Heterodimerization and endocytosis of Arabidopsis brassinosteroid receptors BRI1 and AtSERK3 (BAK1). Plant Cell 16, 3216-3229.

Sager RE, Lee JY. 2018. Plasmodesmata at a glance. J Cell Sci 131, Samuels AL, Giddings TH, Staehelin LA. 1995. Cytokinesis in tobacco BY-2 and root tip cells: a new model of cell plate formation in higher plants. J Cell Biol 130, 1345-1357. 
Scheele U, Holstein SE. 2002. Functional evidence for the identification of an Arabidopsis clathrin light chain polypeptide. FEBS Lett 514, 355-360.

Scheuring D, Viotti C, Kruger F, Kunzl F, Sturm S, Bubeck J, Hillmer S,

Frigerio L, Robinson DG, Pimpl P et al. 2011. Multivesicular bodies mature from the trans-Golgi network/early endosome in Arabidopsis. Plant Cell 23, 3463-3481.

Schindelin J, Arganda-Carreras I, Frise E, Kaynig V, Longair M, Pietzsch T, Preibisch S, Rueden C, Saalfeld S, Schmid B et al. 2012. Fiji: an open-source platform for biological-image analysis. Nat Meth 9, 676-682.

Schneitz K, Hülskamp M, Kopczak SD, Pruitt RE. 1997. Dissection of sexual organ ontogenesis: a genetic analysis of ovule development in Arabidopsis thaliana. Development 124, 1367-1376.

Schneitz K, Hülskamp M, Pruitt RE. 1995. Wild-type ovule development in Arabidopsis thaliana: a light microscope study of cleared whole-mount tissue. Plant J 7, 731-749.

Shah K, Russinova E, Gadella TWJ, Willemse J, De Vries SC. 2002. The Arabidopsis kinase-associated protein phosphatase controls internalization of the somatic embryogenesis receptor kinase 1. Genes Dev 16, 1707-1720.

Shah K, Gadella TW, Jr., van Erp H, Hecht V, de Vries SC. 2001. Subcellular localization and oligomerization of the Arabidopsis thaliana somatic embryogenesis receptor kinase 1 protein. Journal of Molecular Biology 309, 641-655.

Sieburth LE, Meyerowitz EM. 1997. Molecular dissection of the AGAMOUS control region shows that cis elements for spatial regulation are located intragenically. Plant Cell 9, 355-365.

Smyth DR, Bowman JL, Meyerowitz EM. 1990. Early flower development in Arabidopsis. Plant Cell 2, 755-767.

Staehelin LA, Moore I. 1995. The plant Golgi apparatus: structure, functional organization and trafficking mechanisms. Annu. Rev. Plant Physiol. Plant Mol. Biol. 46, 261-288.

Stierhof YD, El Kasmi F. 2010. Strategies to improve the antigenicity, ultrastructure preservation and visibility of trafficking compartments in Arabidopsis tissue. Eur J Cell Biol 89, 285-297.

Teh OK, Shimono Y, Shirakawa M, Fukao Y, Tamura K, Shimada T, HaraNishimura I. 2013. The AP-1 $\mu$ adaptin is required for KNOLLE localization at the cell plate to mediate cytokinesis in Arabidopsis. Plant Cell Physiol 54, 838-847. 


\section{Truernit E, Bauby H, Dubreucq B, Grandjean O, Runions J, Barthelemy J,} Palauqui JC. 2008. High-resolution whole-mount imaging of three-dimensional tissue organization and gene expression enables the study of phloem development and structure in Arabidopsis. Plant Cell 20, 1494-1503.

Tse YC, Mo B, Hillmer S, Zhao M, Lo SW, Robinson DG, Jiang L. 2004. Identification of multivesicular bodies as prevacuolar compartments in Nicotiana tabacum BY-2 cells. Plant Cell 16, 672-693.

Uemura T, Kim H, Saito C, Ebine K, Ueda T, Schulze-Lefert P, Nakano A. 2012. Qa-SNAREs localized to the trans-Golgi network regulate multiple transport pathways and extracellular disease resistance in plants. Proc Natl Acad Sci U S A 109, 1784-1789.

Vaddepalli P, Fulton L, Batoux M, Yadav RK, Schneitz K. 2011. Structurefunction analysis of STRUBBELIG, an Arabidopsis atypical receptor-like kinase involved in tissue morphogenesis. PLoS One 6, e19730.

Vaddepalli P, Fulton L, Wieland J, Wassmer K, Schaeffer M, Ranf S, Schneitz K. 2017. The cell wall-localized atypical $\beta-1,3$ glucanase ZERZAUST controls tissue morphogenesis in Arabidopsis thaliana. Development 144, 2259-2269.

Vaddepalli P, Herrmann A, Fulton L, Oelschner M, Hillmer S, Stratil TF, Fastner A, Hammes UZ, Ott T, Robinson DG et al. 2014. The C2-domain protein QUIRKY and the receptor-like kinase STRUBBELIG localize to plasmodesmata and mediate tissue morphogenesis in Arabidopsis thaliana. Development 141, 4139-4148. Van Damme D, Gadeyne A, Vanstraelen M, Inzé D, Van Montagu MC, De Jaeger G, Russinova E, Geelen D. 2011. Adaptin-like protein TPLATE and clathrin recruitment during plant somatic cytokinesis occurs via two distinct pathways. Proc Natl Acad Sci U S A 108, 615-620.

Viotti C, Bubeck J, Stierhof YD, Krebs M, Langhans M, van den Berg W, van Dongen W, Richter S, Geldner N, Takano J et al. 2010. Endocytic and secretory traffic in Arabidopsis merge in the trans-Golgi network/early endosome, an independent and highly dynamic organelle. Plant Cell 22, 1344-1357.

Wang C, Hu T, Yan X, Meng T, Wang Y, Wang Q, Zhang X, Gu Y, SánchezRodríguez C, Gadeyne A et al. 2016a. Differential regulation of clathrin and its adaptor proteins during membrane recruitment for endocytosis. Plant Physiol 171, 215-229. 
Wang J, Cai Y, Miao Y, Lam SK, Jiang L. 2009. Wortmannin induces homotypic fusion of plant prevacuolar compartments. J Exp Bot 60, 3075-3083.

Wang JG, Feng C, Liu HH, Ge FR, Li S, Li HJ, Zhang Y. 2016b. HAPLESS13mediated trafficking of STRUBBELIG Is critical for ovule development in Arabidopsis. PLoS Genet 12, e1006269.

Wang JG, Li S, Zhao XY, Zhou LZ, Huang GQ, Feng C, Zhang Y. 2013. HAPLESS13, the Arabidopsis $\mu 1$ adaptin, is essential for protein sorting at the transGolgi network/early endosome. Plant Physiol 162, 1897-1910.

\section{Wang L, Li H, Lv X, Chen T, Li R, Xue Y, Jiang J, Jin B, Baluška F, Šamaj J et} al. 2015. Spatiotemporal dynamics of the BRI1 receptor and its regulation by membrane microdomains in living Arabidopsis cells. Mol Plant 8, 1334-1349.

Wu G, Liu S, Zhao Y, Wang W, Kong Z, Tang D. 2015. ENHANCED DISEASE RESISTANCE4 Associates with CLATHRIN HEAVY CHAIN2 and Modulates Plant Immunity by Regulating Relocation of EDR1 in Arabidopsis. Plant Cell

Yadav RK, Fulton L, Batoux M, Schneitz K. 2008. The Arabidopsis receptor-like kinase STRUBBELIG mediates inter-cell-layer signaling during floral development. Dev Biol 323, 261-270.

\section{Zhou J, Liu D, Wang P, Ma X, Lin W, Chen S, Mishev K, Lu D, Kumar R,} Vanhoutte I et al. 2018. Regulation of Arabidopsis brassinosteroid receptor BRI1 endocytosis and degradation by plant U-box PUB12/PUB13-mediated ubiquitination. Proc Natl Acad Sci U S A 115, E1906-E1915. 


\section{Tables}

Table 1. Number of periclinal cell divisions in the L2 layer of stage 3 floral meristems.

\begin{tabular}{|l|l|l|l|}
\hline Genotype & $\mathrm{NPCD}^{\mathrm{a}}$ & Percentage & $\mathrm{NFM}^{\mathrm{b}}$ \\
\hline Col & 12 & 17.6 & 68 \\
\hline sub-9 & 17 & 36.2 & 47 \\
\hline chc1-1 & 6 & 23.1 & 26 \\
\hline chc1-2 & 7 & 20.0 & 35 \\
\hline chc2-1 & 7 & 22 & 31 \\
\hline chc2-2 & 5 & 22.5 & 25 \\
\hline chc1-1 sub-9 & 6 & 20.0 & 30 \\
\hline chc1-2 sub-9 & 7 & 19.4 & 36 \\
\hline chc2-1 sub-9 & 7 & 25.9 & 27 \\
\hline chc2-2 sub-9 & 7 & 18.9 & 37 \\
\hline
\end{tabular}

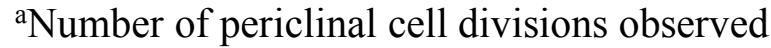

${ }^{b}$ Number of floral meristems observed

Table 2. Comparison of integument defects between sub-9, chc and chc sub-9 mutants.

\begin{tabular}{|l|l|l|l|}
\hline Genotype & N total & N with defects & Percentage \\
\hline Col & 274 & 0 & 0 \\
\hline sub-9 & 291 & 82 & 28.2 \\
\hline chc1-1 & 130 & 0 & 0 \\
\hline chc1-2 & 121 & 0 & 0 \\
\hline chc2-1 & 126 & 0 & 0 \\
\hline chc2-2 & 230 & 0 & 0 \\
\hline chc1-1 sub-9 & 235 & 14 & 6 \\
\hline chc1-2 sub-9 & 185 & 11 & 6 \\
\hline chc2-1 sub-9 & 211 & 14 & 6.6 \\
\hline chc2-2 sub-9 & 237 & 13 & 5.5 \\
\hline
\end{tabular}




\section{Figure Legends}
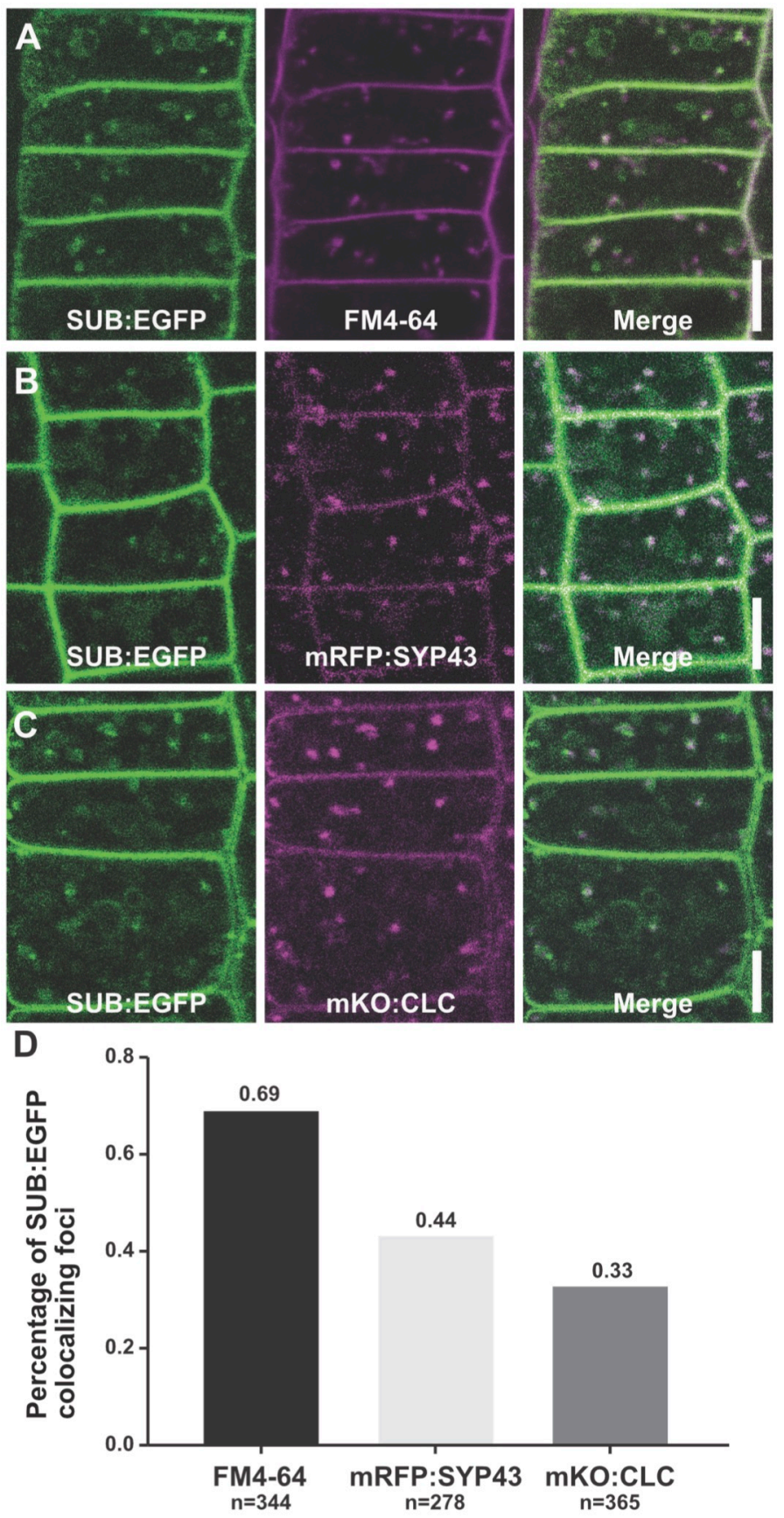

Figure 1. Sub-cellular localization of SUB:EGFP. Fluorescence micrographs show optical sections of epidermal cells of root meristems of five to six days-old seedlings. (A) Partial colocalization of SUB:EGFP and FM4-64 foci upon treating cells with FM4-64 for five minutes. (B) Partial colocalization of SUB:EGFP and mRFP:SYP43 puncta. (C) Partial colocalization of SUB:EGFP and mKO:CLC signals. (D) Quantitative colocalization analysis of SUB:EGFP-positive foci and reporter signals shown in A, B and C. $n$, total number of analyzed SUB:EGFP foci. Scale bars: $5 \mu \mathrm{m}$. 

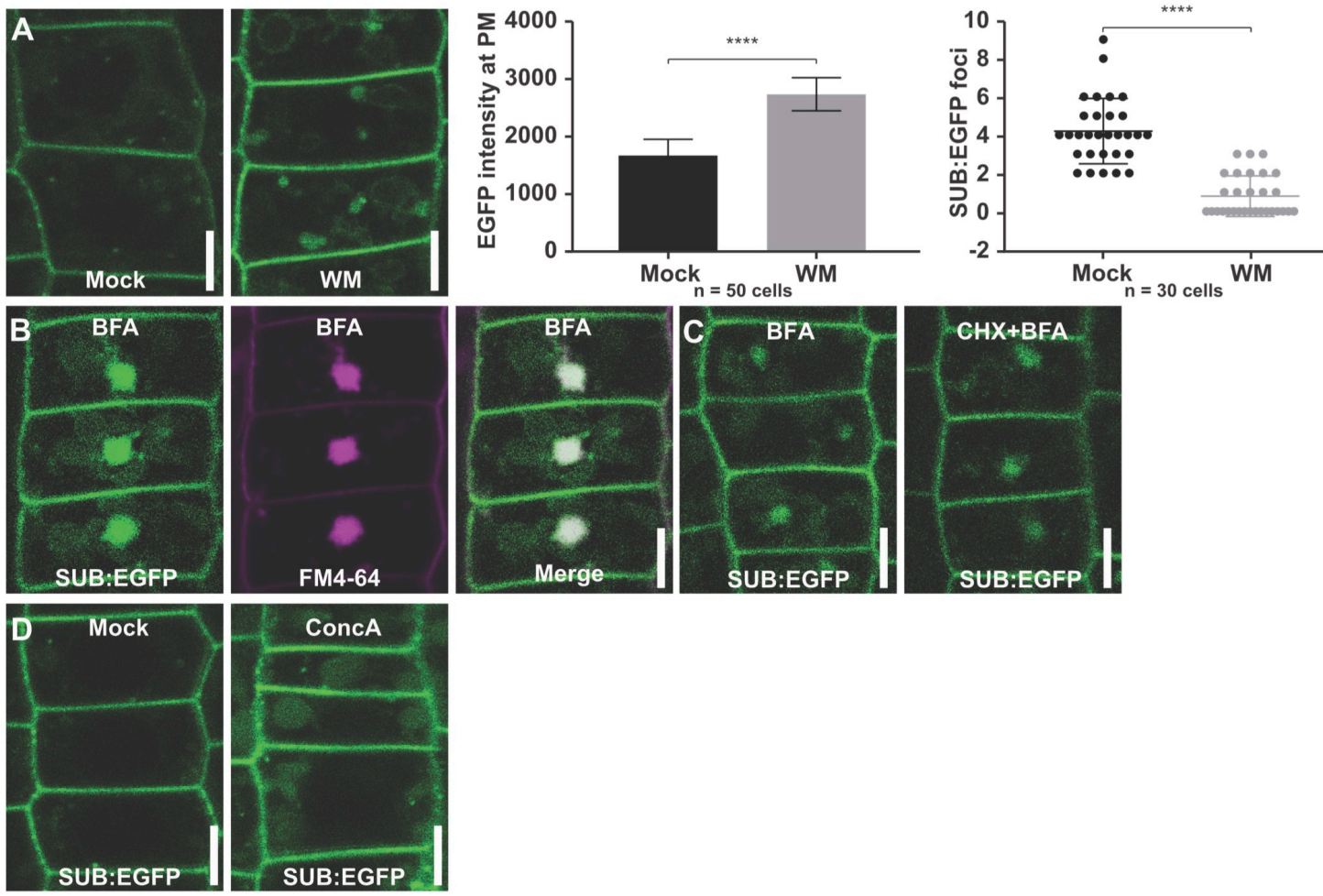

Figure 2. Sub-cellular localization of SUB:EGFP upon drug treatments.

Fluorescence micrographs show optical sections of epidermal cells of root meristems of five to six days-old seedlings. (A) Subcellular localization of SUB:EGFP signal in the presence of Wortmannin and DMSO (mock control) (left). Graphs represent quantification of the EGFP intensity at plasma membrane (middle panel, $\mathrm{n}=50$ cells across six roots) and the number of SUB:EGFP-positive endosomes per cell (right panel, $\mathrm{n}=30$ cells across six roots) after incubation. Asterisks represent statistical significances $(\mathrm{P}<0.0001)$ as judged by Student's $t$ test. (B) SUB:EGFP signal is detected in BFA bodies upon BFA treatment. (C) SUB:EGFP signal is detected in BFA compartments in the presence of CHX. (D) SUB:EGFP signal is observed in lytic vacuoles after ConcA treatment. Abbreviations: ROI, region of interest. Scale bars: $5 \mu \mathrm{m}$. 


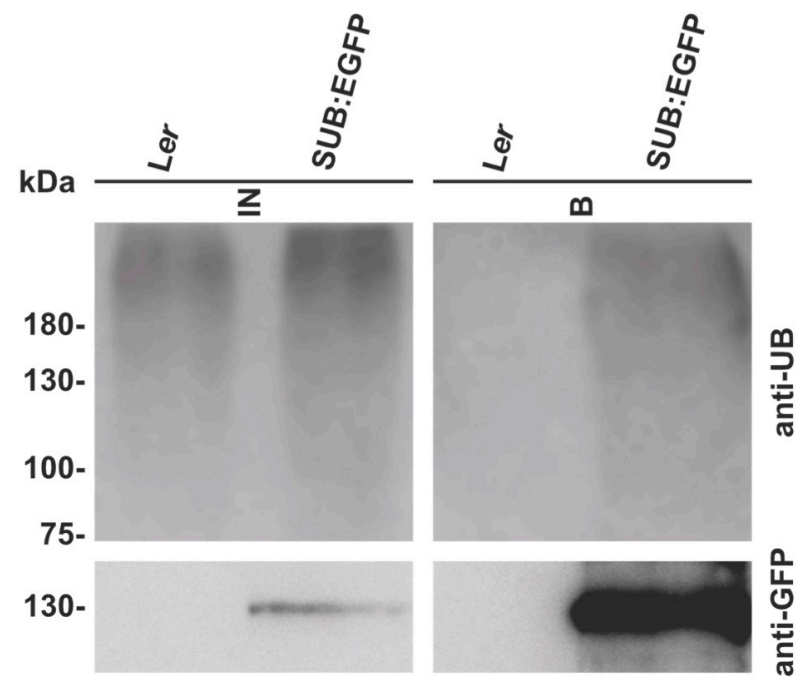

Figure 3. In vivo ubiquitination of SUB. Western blot analysis of

immunoprecipitates obtained from wild type (Ler) and sub-1 pUBQ::gSUB:EGFP lines are shown. Immunoprecipitation was performed using an anti-GFP antibody. Immunoblots were probed with the P4D1 anti-Ub antibody (top panel) and an antiGFP antibody (bottom panel). Abbreviations: B: bound fraction; IN, input.

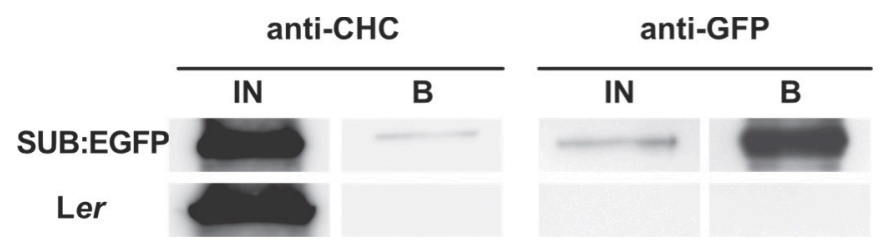

Figure 4. Co-immunoprecipitation of CHC with SUB:EGFP. Total extracts of seven day-old SUB:EGFP-expressing seedlings (upper panel) or wild-type seedlings (lower panel) were immunoprecipitated using GFP-Trap MA beads. Immunoblots were probed with anti-CHC (left panel) or anti-GFP antibodies (right panel).

Abbreviations: B: bound fraction; IN, input. 

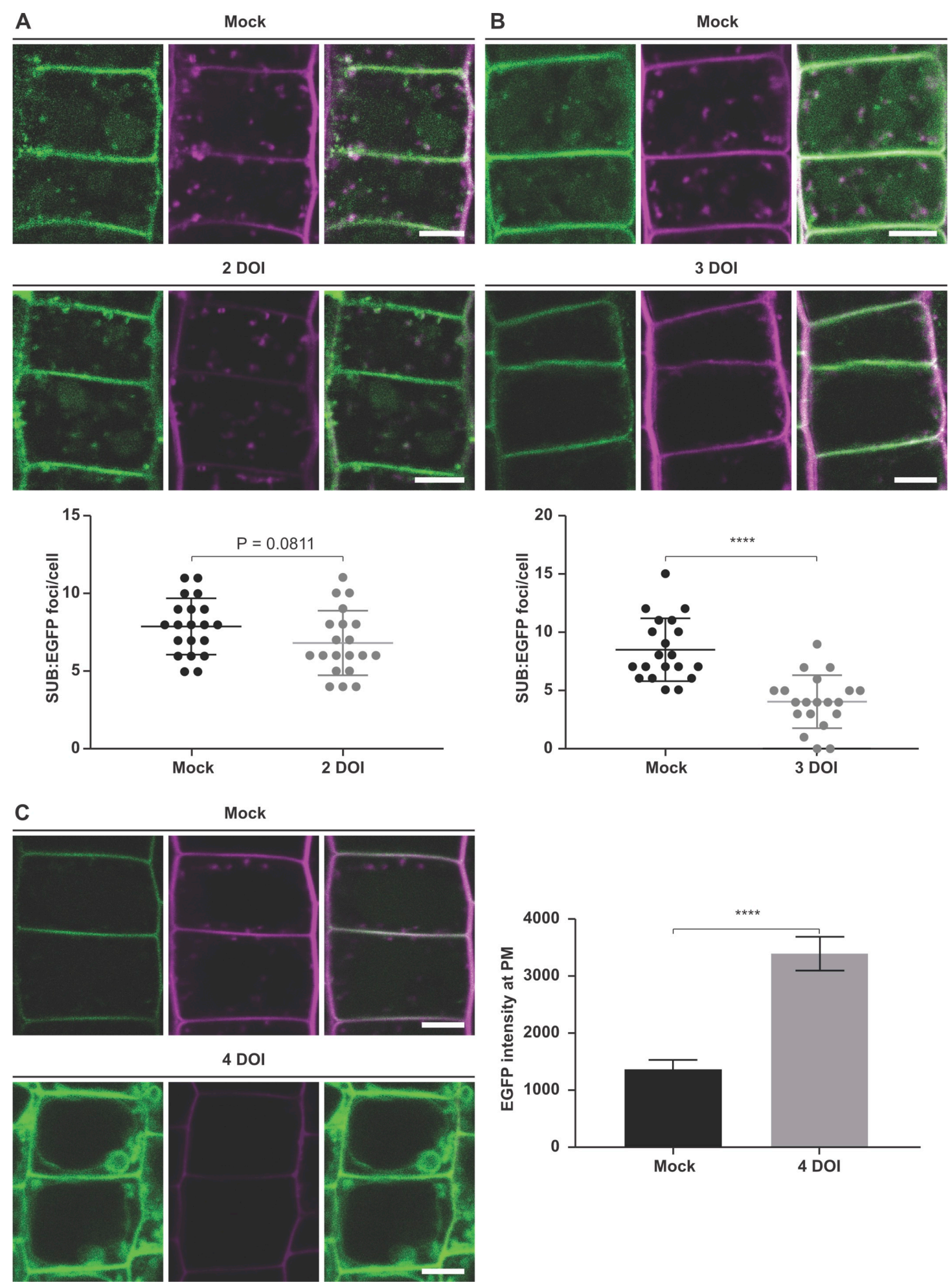

4 DOI
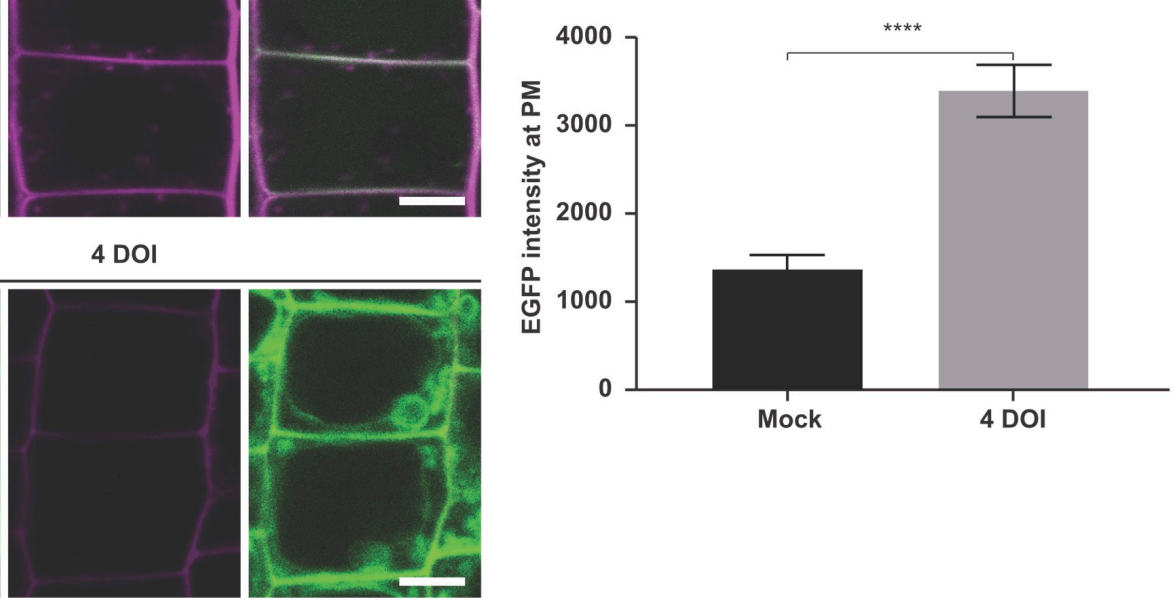

Figure 5. Requirement of clathrin function for SUB endocytosis. Fluorescence

micrographs show optical sections of epidermal cells of root meristems. (A) to (C) Internalization of SUB:EGFP and uptake of endocytic tracer dye FM4-64 in epidermal meristems cells of three days-old INTAM $>>$ RFP-CHC1 (HUB1)/pUBQ::SUB:EGFP seedlings that were placed on $2 \mu \mathrm{M} 4-$ hydroxytamoxifen-containing induction medium for two, three, or four days, 
respectively. Ethanol served as mock. Graphs represent quantification of the number of SUB:EGFP-positive spots per cell (A, B) and of the EGFP intensity at plasma membrane (C) after incubation. Abbreviation: DOI, days on induction medium. Scale bars: $5 \mu \mathrm{m}$.
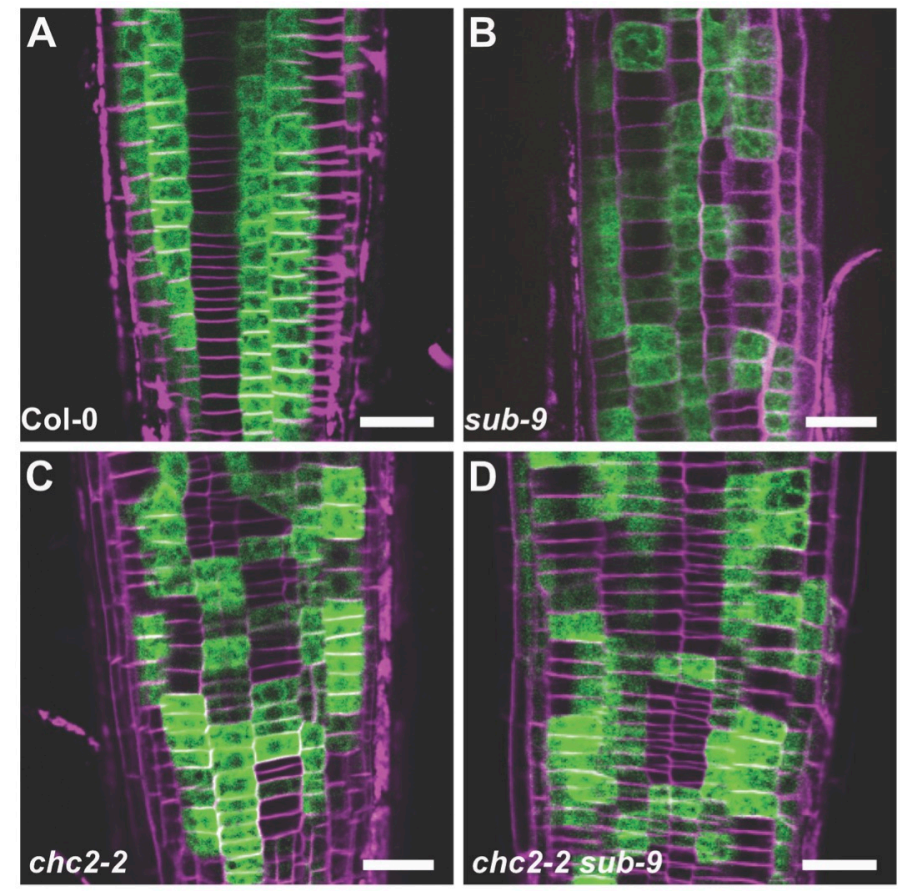

Figure 6. Expression pattern of the pGL2::GUS:EGFP reporter in $c h c 2-2$ and

chc2-2 sub-9 mutants. Fluorescence micrographs show optical sections of epidermal cells of root meristems of seven days-old seedlings. FM4-64 was used to label cell outlines. (A) Col-0. (B) sub-9. (C) chc2-2. (D) chc2-2 sub-9. Note the similarly altered pattern in (B) to (D). Scale bars: $25 \mu \mathrm{m}$. 

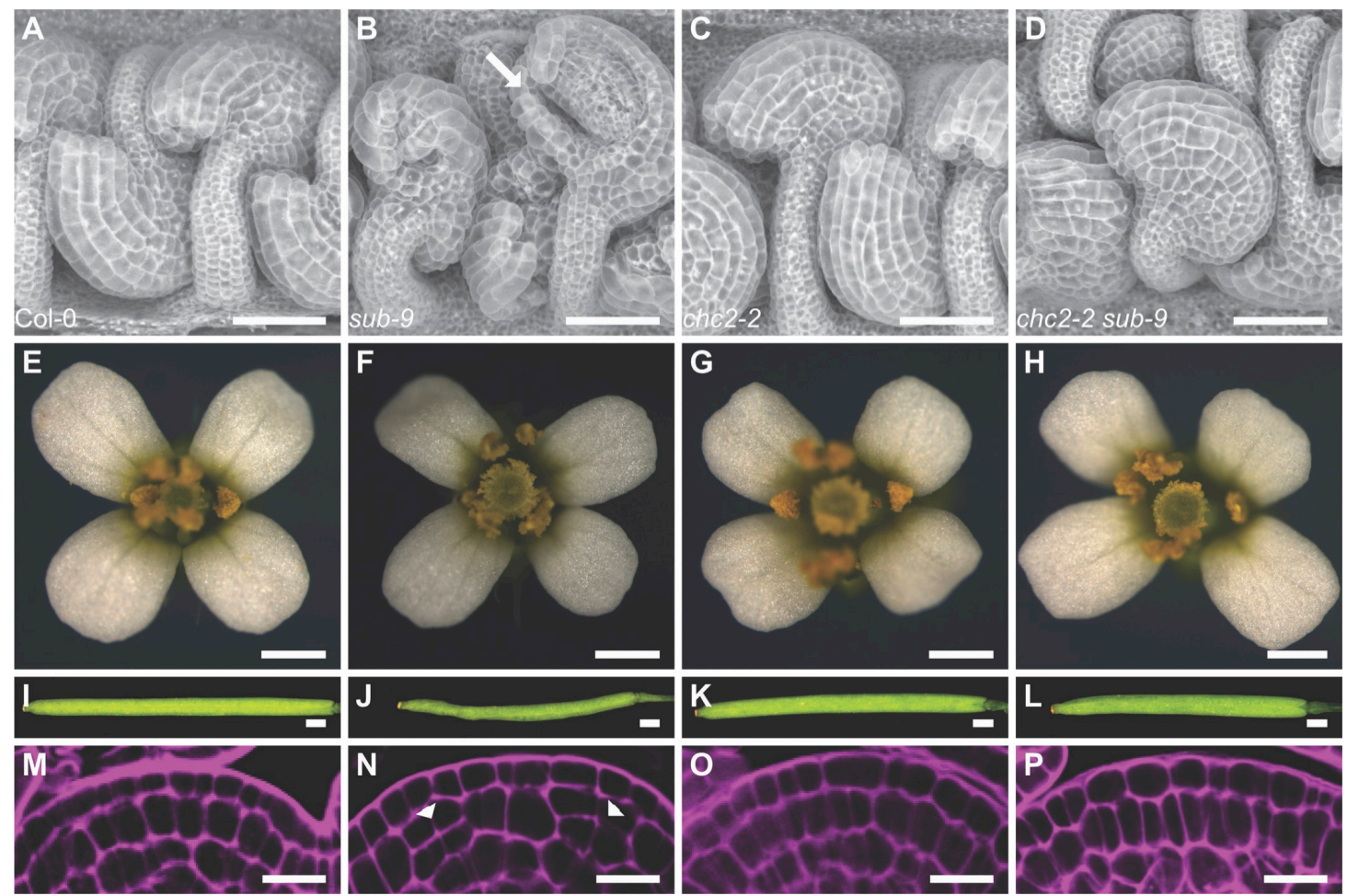

Figure 7. Phenotype comparison between Col-0, sub-9, chc2-2 and chc2-2 sub-9.

(A) to (D) Scanning electron micrographs of stage 4 ovules (stages according to (Schneitz et al., 1995)). (B) Note the aberrant outer integument (arrow). (E) to (H) Morphology of mature stage 13 or 14 flowers (stages according to (Smyth et al., 1990)). (I) to (L) Morphology of siliques. (M) to (P) Central region of stage 3 floral meristems stained with pseudo-Schiff propidium iodide (mPS-PI). (N) Arrowheads indicate aberrant cell division planes. (P) Note the defects of the $s u b-9$ phenotype were partially rescued in chc sub-9 double mutants. Scale bars: (A) to (D) $50 \mu \mathrm{m}$, (E) to $\mathbf{( H )} 0.5 \mathrm{~mm}$, (I) to (L) $1 \mathrm{~mm}, \mathbf{( M )}$ to $(\mathbf{P}) 10 \mu \mathrm{m}$. 